\title{
PALEODIETAS EN RESTOS HUMANOS DEL BOSQUE MERIDIONAL DE NEUQUÉN, PATAGONIA ARGENTINA
}

ALBERTO E. PÉREZ ${ }^{\mathrm{a}}$, AUGUSTO TESSONE ${ }^{\mathrm{b}} \&$ JOSÉ LUIS LANATA ${ }^{c}$

\section{RESUMEN}

Se presentan tres novedosos registros de isótopos de carbono y nitrógeno procedentes de dos contextos funerarios a cielo abierto en el interior del bosque norpatagónico. Las muestras humanas analizadas confirman la utilidad de la técnica para diferenciar las dietas terrestres de las poblaciones humanas del área andina de Patagonia. Los resultados reflejan la importancia del bosque y sus recursos en el último milenio, fortaleciendo los modelos que plantean la idea de poblaciones humanas explotando de manera regular y permanente el bosque y sus recursos en la región andina oriental de Patagonia septentrional.

PALABRAS CLAVE: isótopos estables, paleodieta, bosque norpatagónico.

\section{PALEODIETS IN HUMAN REMAINS OF THE SOUTHERN FOREST OF NEUQUÉN, PATAGONIA ARGENTINA}

\begin{abstract}
Three novel records of carbon and nitrogen isotopes are presented from two open-air funerary contexts in the interior of the North Patagonian forest. The human samples analyzed confirm the usefulness of the technique to differentiate terrestrial diets from human populations in the Andean area of Patagonia. The results reflect the importance of the forest and its resources in the last millennium, strengthening the models suggesting human populations regularly and permanently exploiting the forest and its resources in the eastern Andean region of northern Patagonia.

KEY WORDS: Stable isotopes, paleodiet, North Patagonian forest.

a Departamento de Antropología. Universidad Católica de Temuco. Manuel Montt 56 (4780000), Temuco, Chile. $\measuredangle$ aperez@uct.cl

b Instituto de Geocronología y Geología Isotópica, CONICET-UBA, Ciudad Autónoma de Buenos Aires (C1428EHA), Argentina. gutitessone@gmail.com

c IIDyPCa, CONICET-UNRN, Mitre 630, (8400) Bariloche, Argentina. jllanata@conicet.gov.ar
\end{abstract}




\section{INTRODUCCIÓN}

La idea del bosque andino patagónico como un potencial hábitat para la ocupación humana ha comenzado un proceso importante de discusión durante la última década (Arrigoni \& Fernández, 2004; Borrero, 2005; Hajduk et al. 2008; Pérez \& Smith, 2008; Lezcano et al. 2010; Fernández et al. 2013). Recientemente, Bellelli et al. (2018) han agrupado las diferentes posturas en tres modelos diferentes: a- los que postulan que las poblaciones permanecen más tiempo en la estepa y el ecotono que en el bosque; b- los que sugieren poblaciones residentes en forma semipermanente en el bosque integrando el ecotono de manera regular, y c- los que postulan la existencia de grupos adaptados al bosque. Es importante aclarar que estos tres diferentes modelos pueden ser reflejo de las particularidades de cada sector de bosque y, por lo tanto, no son necesariamente antagónicos (Pérez, 2010; Fernández et al. 2011). En el marco de esta discusión se presentan valores $\delta^{13} \mathrm{C}$ y $\delta^{15} \mathrm{~N}$ correspondientes a individuos recuperados en sitios arqueológicos del bosque andino patagónico en la región meridional de Neuquén, Argentina. Los isótopos estables del carbono y nitrógeno medidos en diversos tejidos registran el consumo de recursos por parte de los individuos. El desarrollo de estos trabajos en la arqueología de Patagonia ha permitido diferenciar los recursos de la estepa de los obtenidos del bosque, reflejándose en diferentes especies, incluyendo restos humanos. Fernández y Tessone (2014) han demostrado la importancia de esta metodología para discutir los modelos de uso del bosque en el área septentrional de Patagonia. En esta oportunidad se presentan valores de tres individuos recuperados en dos entierros del sur de Neuquén: Siete Manzanos y Newen Antug (Fig. 1), ambos con fechas correspondientes a los últimos 1.000 años. El objetivo de este informe es dar a conocer nuevos valores isotópicos de restos humanos y colaborar en la discusión sobre el uso del bosque y sus recursos en Patagonia norte por parte de las poblaciones humanas.

Modelo regional para

el sur de Neuquén, Argentina

Los Bosques Templados Andinos Norpatagónicos son los ambientes de mayor productividad ambiental de la Patagonia continental (Paruelo et al. 1998), y su potencialidad alimenticia es muy importante para los humanos según registros etnohistóricos y etnográficos (Aldunate \& Villagrán, 1992; Gallardo \& Mege, 2012; Skewes \& Guerra, 2016). Los postulados de adaptación boscosa surgen de hacer extensiva a la vertiente oriental cordillerana la distribución territorial de las poblaciones históricas Reche-Mapuche (Aldunate, 1996) y sus antecesores, cuyo correlato arqueológico es conocido por su alfarería como Tradición Bícroma Rojo sobre Blanco (Complejo El Vergel y estilo Valdivia, siglos IX a XIX) y, más tempranamente, como Complejo Pitrén (siglos IV a $\mathrm{XI}$. Estos complejos arqueológicos incluyen una expresión cordillerana tardía denominada Tradición Arqueológica de los Bosques Templados del centro sur de Chile (hasta el siglo XIV), y que ocupa ambas vertientes de la cordillera andina. Recientemente han sido caracterizados como períodos Alfareros Tardío y Temprano respectivamente (Adán et al. 2016), cuya distribución oriental hemos hecho extensiva, en base a características compartidas del registro arqueológico, a las cuencas lacustres y boscosas de los lagos Lácar y Meliquina (Pérez, 2010, 2016, 2018a).

El área de estudio incluye sitios arqueológicos emplazados en la cuenca del río Valdivia, sector Oriental Cordillerano (Pérez, 2016), parte del Departamento Lácar, provincia del Neuquén, Argentina. A partir del relevamiento de 36 sitios arqueológicos, se distinguieron ocupaciones pertenecientes a un Período Alfarero Temprano y otros a un Período Alfarero Tardío, incorporando así, y solo con fines comparativos, las clasificaciones de investigadores trasandinos a partir de reconocer una continuidad entre el paisaje y la cultura material a lo largo de la cuenca hidrográfica del río Valdivia (Pérez, 2016). El Período Alfarero Temprano se caracteriza por pequeños grupos familiares con un sistema de subsistencia cazador-recolectorpescador-horticultor y movilidad estacional de tipo residencial, emplazándose predominantemente en las riberas de lagos y ríos, y en zonas precordilleranas lacustres (Aldunate, 1989; Adán et al. 2016). $\mathrm{Su}$ dieta estuvo centrada en la recolección de alimentos vegetales, complementada con caza y pesca de fauna típica del ámbito boscoso y lacustre, y horticultura de tala y roza. En suma, 


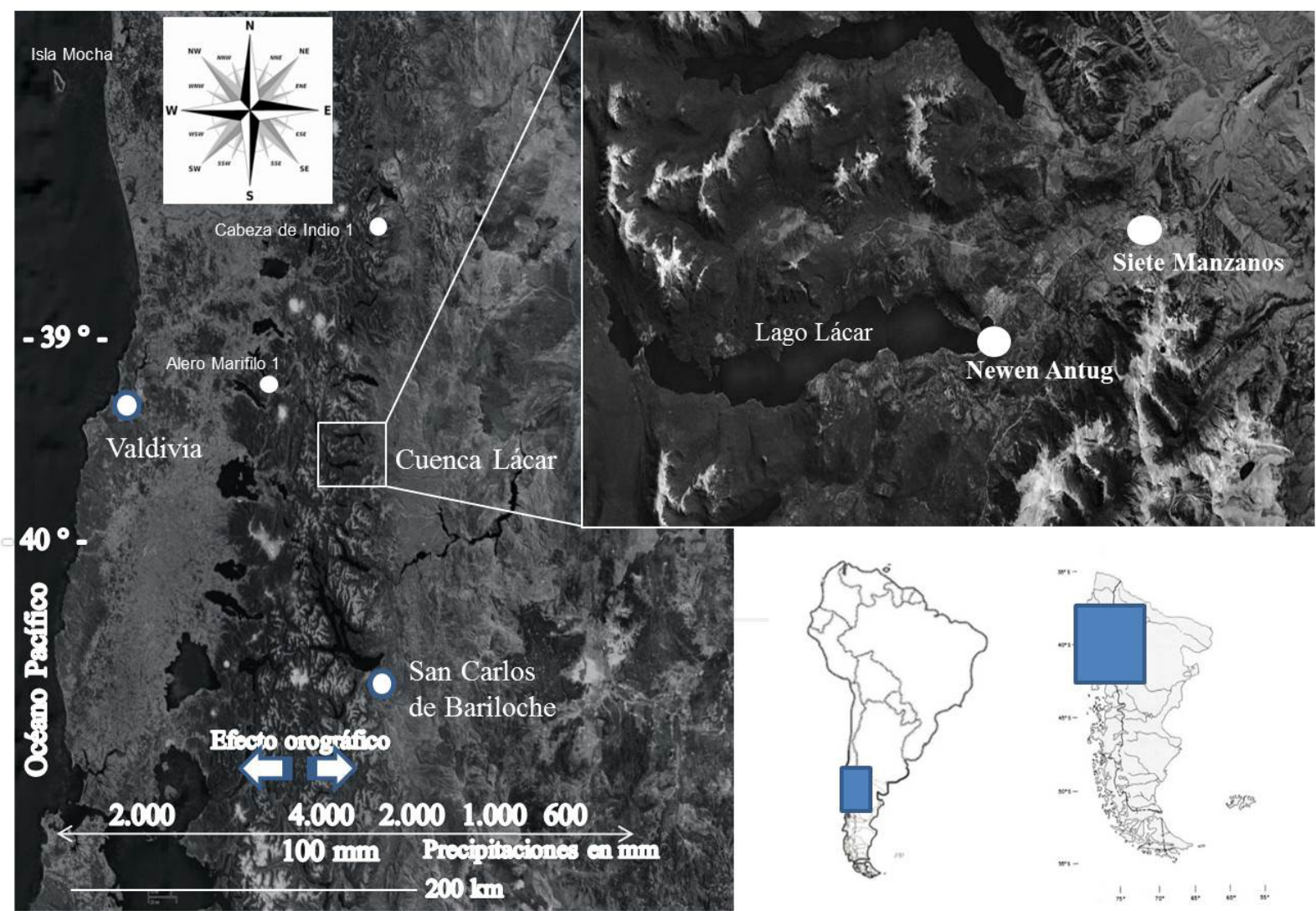

Fig. 1. Ubicación de sitios Newen Antug y Siete Manzanos.

una estrategia adaptativa boscosa y lacustre (Adán et al. 2010; Adán \& Mera, 2011; Navarro et al. 2011). El Período Alfarero Tardío muestra también gran representación en la cuenca del lago Lácar. Se postula su comienzo en el siglo XI y su mayor presencia en los valles centrales trasandinos con límite en el corredor Mahuidanche-Lastarria, y con dataciones en sitios orientales cordilleranos como Newen Antug entre 1.040 y 880 años AP (Pérez, 2016) y 480 años AP en Los Radales 1 (Pérez, 2018b). La economía estaría centrada en la recolección vegetal estacional, complementada con caza, pesca y posiblemente horticultura/ agricultura, y domesticación de animales en escalas que aún desconocemos. Espacios productivos de estas poblaciones han sido sugeridos en sitios continentales de los valles centrales (Purén y Lumaco) y costa (lago Budi) e insulares como Isla Mocha (Adán et al. 2016). Sin embargo, las actividades extractivas seguirían siendo fundamentales entre la costa y el primer cordón cordillerano de la costa y espacios cordilleranos lacustres andinos (Pérez et al. 2013; Pérez, 2016).

\section{Contextos funerarios}

La presencia de restos humanos en ambientes de bosque es escasa y mayormente representada por sitios arqueológicos bajo reparos rocosos (Rizzo, 2017). Los individuos que analizamos provienen de dos contextos funerarios a cielo abierto distintos ubicados en la ladera norte del cordón Chapelco (Fig. 1), dentro de bosque subantártico de Nothofagus (Armesto et al. 1996; Donoso, 1993). Este bosque está conformado por especies como radal (Lomathia hirsuta), maitén (Maytenus magallanica), ñire (Nothofagus antarctica), lenga (Nothofagus pumilla), roble (Nothofagus oblicua), entre otras; pero antropizado, y donde la vegetación predominante es el ciprés de la cordillera (Austrocedrus chilensis). Los materiales analizados provienen de 
actividades de rescate realizadas por el laboratorio de Arqueología y Etnohistoria de la Municipalidad de San Martín de los Andes, Neuquén, entre 2012 y 2015.

Por un lado, el sitio Siete Manzanos $\left(40^{\circ} 08^{\prime} 14^{\prime \prime} \mathrm{S}-71^{\circ} 13^{-4} 45^{\circ-} \mathrm{O}\right)$ se ubica en el sector oriental de la ladera norte del cordón Chapelco, conservando un pequeño parche relictual de manzanos (Malus sp.) centenarios (Fig. 2, individuo $1)$. Es un sitio a cielo abierto de actividades múltiples asociado a artefactos líticos y cerámicos correspondientes a las expresiones cordilleranas tardías del Período Alfarero Temprano de la secuencia regional (Pérez, 2016). En este caso se trata de un individuo femenino mayor de 35 años, inhumado en forma directa hace $850 \pm 60$ años AP (LP 3271, hueso humano), sin ajuar asociado al cuerpo.

Por otro lado, el sitio arqueológico Newen Antug (40 09-44“-S -71 $\left.20^{\circ} 49^{--} \mathrm{O}\right)$ se encuentra en proximidad del lago Lácar, sobre la ladera norte del mismo cordón Chapelco. Es un sitio a cielo abierto de carácter multicomponente que presenta una antigüedad de $1.040 \pm 50$ años AP (LP 3411, artefacto de madera asociado a restos humanos). Ocupado como sitio habitacional de actividades múltiples y espacio formal de entierros humanos desde esta fecha y luego entre los $880 \pm 40$ años AP (LP 3426, hueso humano) y $540 \pm 50$ años AP (LP 3024 , carbón vegetal). Si bien otro sector del lugar fue sistemáticamente saqueado desde hace décadas por lugareños, se recuperaron dos individuos en contexto primario, correspondientes a un individuo femenino de aproximadamente 50 años de edad y otro masculino de aproximadamente 35 años. Los entierros presentaron ajuares cerámicos de la Tradición Bícroma Rojo sobre Blanco o Período Alfarero Tardío (Fig. 2, individuos 2 y 3), artefactos líticos que incluyen cabezales pedunculados pequeños de obsidiana del grupo químico Yuco, obtenidos en sectores boscosos siempreverdes de la costa norte del lago Lácar. Entre los conjuntos óseos identificados se destaca la presencia de la almeja de agua dulce (Diplodon chilensis) y fauna terrestre como cérvidos (Hippocamelus bisulcus), utilizados tanto como alimento, tecnología (retocador) y parte del ajuar funerario. Se realizaron múltiples fechados del sitio, pero los cuerpos analizados para este trabajo son los asociados a la datación radiocarbónica de $540 \pm 50$ años AP (LP 3024, carbón vegetal) de madera carbonizada en asociación directa a los cuerpos.
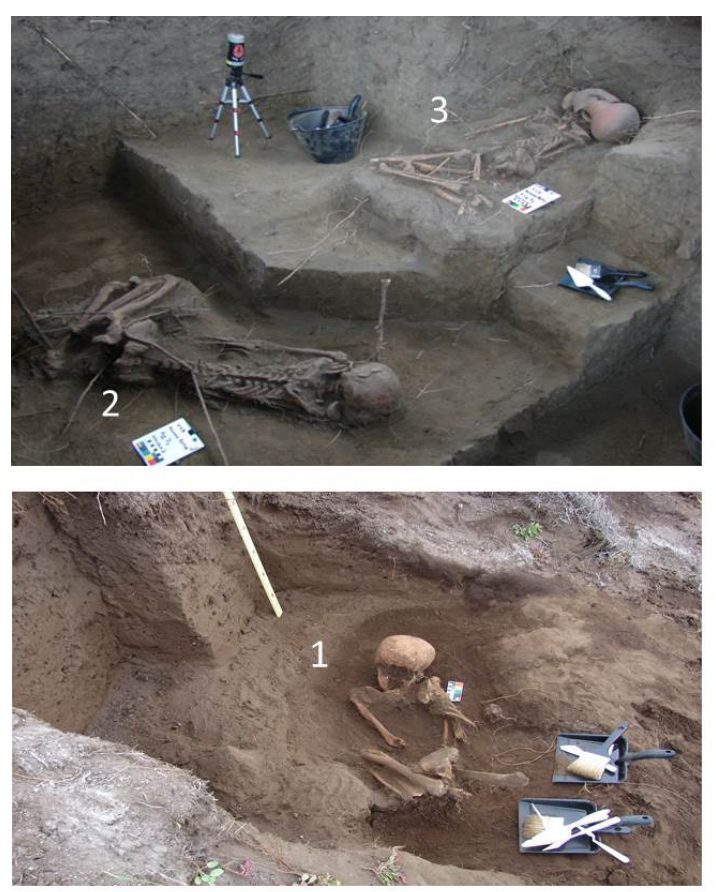
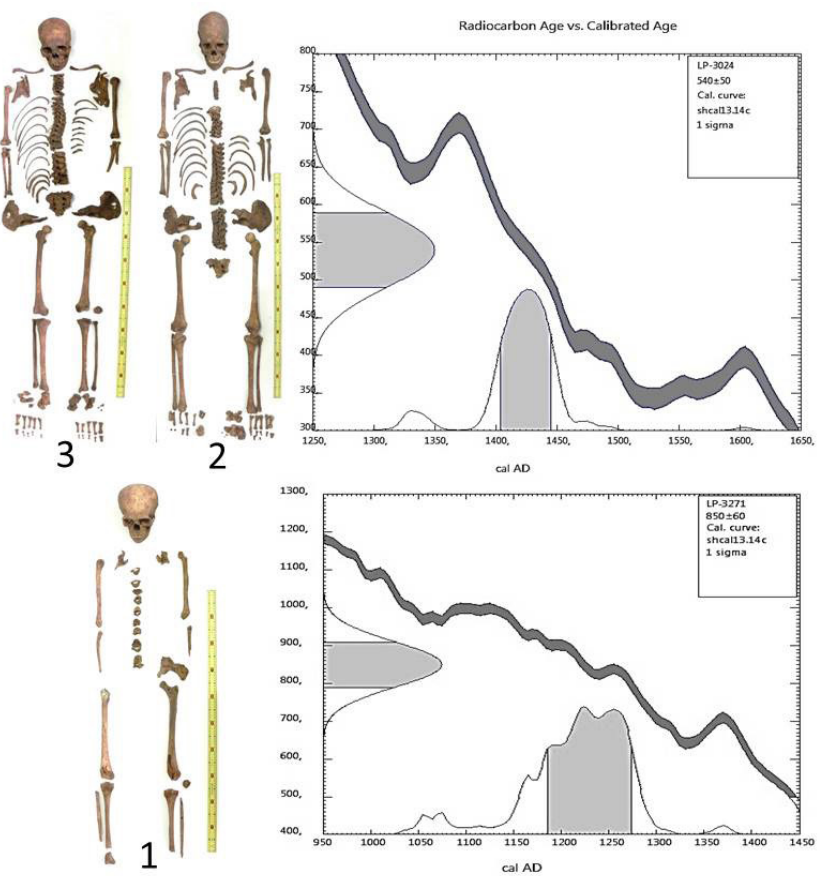

Fig. 2. 1- Siete Manzanos, 2 y 3- Newen Antug. Disposición de los cuerpos y dataciones ${ }^{14} \mathrm{C}$. 


\section{Dietas y Recursos de bosque}

Se presentan valores $\delta^{13} \mathrm{C}$ y $\delta^{15} \mathrm{~N}$ sobre el colágeno de tres individuos. La extracción de colágeno y su medición en un espectrómetro de masas se realizó en el Instituto de Geocronología y Geología Isotópica (INGEIS-CONICET-UBA). Al ser mediciones sobre la proteína ósea, los valores reflejarán principalmente el consumo de recursos proteicos, principalmente animales. De esta manera, la presente información será utilizada para evaluar el origen y el tipo de proteína consumida por los individuos durante aproximadamente los últimos 10 años de vida.

Respecto al estado diagénesis $y$ contaminación, la muestra AIE 36880 procedente del individuo femenino de Newen Antug presenta una mala relación $\mathrm{C} / \mathrm{N}$, con un valor de 3,8 por fuera del rango normal $(2,9 / 3,6)$. Las restantes dos muestras provenientes del individuo masculino del mismo sitio arqueológico y del femenino del sitio Siete Manzanos, se encuentran dentro del rango normal, por lo que se considera que sus valores son primarios (Tabla 1). En el análisis que sigue a continuación se considerarán solamente estas últimas dos muestras. Las mismas presentan valores homogéneos en ambos isótopos estables, con valores de $-20,9 \%$ y $-20,7 \%$ o para el $\delta^{13} \mathrm{C}$ y de $7.0 \%$ o y $7.8 \%$ o para el $\delta^{15} \mathrm{~N}$ (Tabla 1 ). Es importante destacar que la muestra descartada registra valores similares en ambos isótopos. En términos del $\delta^{13} \mathrm{C}$, los valores reflejan el consumo de recursos pertenecientes a cadenas tróficas $\mathrm{C}_{3}$. Algo esperable en el ambiente de Patagonia donde esta vía fotosintética es dominante. Por su parte, el $\delta^{15} \mathrm{~N}$ registra el consumo de herbívoros.

$\mathrm{Si}$ bien no se dispone de una ecología isotópica para el área, Fernández y Tessone (2014) han desarrollado una serie de mediciones de los principales recursos faunísticos del norte de Patagonia (Tabla 2). En este trabajo se registraron claramente las diferencias entre los sectores de bosque y estepa (ver también Barberena et al. 2011), tanto en herbívoros de gran porte (i.e. guanaco y huemul) como en roedores (Ctenomys y Caviidae). De esta manera, se utilizará este marco de referencia (Fernández \& Tessone, 2014) para profundizar las interpretaciones de estos individuos del sur de Neuquén. En este sentido, las dos muestras analizadas registran una dieta que se relaciona claramente con proteínas provenientes de bosque. Esto se aprecia en la Fig. 3, en donde se comparan los valores obtenidos junto a la ecología isotópica construida por Fernández y Tessone (2014). Para facilitar su lectura se estimó la dieta teórica a partir de considerar una discriminación isotópica de $1 \%$ y $4 \%$ para el $\delta^{13} \mathrm{C}$ y $\delta^{15} \mathrm{~N}$. Al igual que las presentadas en su oportunidad por Fernández y Tessone (2014), aquí también las paleodietas de los individuos permiten plantear un

Tabla 1. Resultados $\mathrm{d}^{13} \mathrm{C}, \mathrm{d}^{15} \mathrm{~N}$ y $\mathrm{C} / \mathrm{N}$ en muestras humanas de Siete Manzanos y Newen Antug.

\begin{tabular}{lcccccc}
\hline Procedencia & Sexo & Edad & AIE & $\delta^{13} \mathrm{C} \%$ ov V-PDB & $\delta^{15} \mathrm{~N}$ \%ovs AIR & $\mathrm{C} / \mathrm{N}$ \\
\hline \multirow{2}{*}{ Newen Antug } & masculino & 35 & 36879 & $-20,7$ & 7,8 & 3,3 \\
\multirow{2}{*}{ Siete Manzanos } & femenino & 50 & 36880 & $-21,3$ & 6,9 & 3,8 \\
& femenino & $>35$ & 36881 & $-20,9$ & 7,0 & 3,4 \\
\hline
\end{tabular}

Tabla 2. Valores $\mathrm{d}^{13} \mathrm{C}$ y d $\mathrm{d}^{15} \mathrm{~N}$ de recursos animales de Patagonia Norte (tomado de Fernández \& Tessone, 2014).

\begin{tabular}{lcccccccc}
\hline & \multicolumn{2}{c}{ Caviidae } & \multicolumn{2}{c}{ Ctenomys } & \multicolumn{3}{c}{ Hippocamelus bisulcus } & \multicolumn{2}{c}{ Lama guanicoe } \\
& $\mathrm{d}^{13} \mathrm{C}$ & $\mathrm{d}^{15} \mathrm{~N}$ & $\mathrm{~d}^{13} \mathrm{C}$ & $\mathrm{d}^{15} \mathrm{~N}$ & $\mathrm{~d}^{13} \mathrm{C}$ & $\mathrm{d}^{15} \mathrm{~N}$ & $\mathrm{~d}^{13} \mathrm{C}$ & $\mathrm{d}^{15} \mathrm{~N}$ \\
\hline $\mathrm{N}$ & 4 & 4 & 3 & 3 & 4 & 4 & 5 & 5 \\
Media & -22.3 & 1.7 & -21.5 & 1.4 & -21.2 & 2.2 & -20.3 & 6.3 \\
Desvío Estándar & 1.0 & 0.6 & 0.1 & 0.7 & 1.2 & 1.3 & 1.0 & 1.3 \\
Minino & -23.2 & 1.1 & -21.6 & 1.0 & -23.0 & 0.4 & -21.4 & 5.01 \\
Máximo & -21.0 & 2.4 & -21.5 & 2.3 & -20.4 & 3.5 & -19.3 & 8.2 \\
\hline
\end{tabular}


uso de recursos restringido -o mayoritariamentede bosque, tal como lo plantea el modelo C (Bellelli et al. 2018).

A nivel regional, se observa una clara diferencia con los valores registrados en el centro y norte de Neuquén del sector de la estepa (Gordón et al. 2017; Barberena et al. 2018). A su vez, se registraron diferencias con los valores informados para los restos humanos recuperados en Isla Mocha, Chile, los cuales también fueron asignados a ocupaciones costeras, habitacionales y funerarias del Complejo Pitrén y El Vergel. Éstos presentan consumo de recursos marinos, aunque no en forma mayoritaria (Campbell et al. 2017). Por último, en términos supra-regionales, nuestras muestras se diferencian significativamente de las recuperadas en la estepa, como las del lago Salitroso en Santa Cruz (Tessone, 2010) o las restantes muestras de Patagonia continental (Barberena, 2002; Borrero \& Barberena, 2006; Flensborg et al. 2018; Zilio et al. 2018). En suma, las paleodietas de restos óseos humanos recuperados en el bosque del norte de Patagonia continental presentan valores particulares que hacen referencia a un uso del bosque y sus recursos de manera predominante.

Asimismo, en referencia al modelo de subsistencia, este resultado permite confirmar los postulados de Pérez y Smith (2008) y Pérez (2010), correspondientes con las expectativas de sitios arqueológicos de "interior de bosque", donde el registro sugiere que el área de captación diaria de los recursos del sitio es mayoritaria, cuando no exclusivamente, boscosa (Pérez, 2010). A la presencia de huemul en Newen Antug (Pérez $\&$ Reyes, 2009), se suma el uso alimenticio de huemul y pudú junto a otra fauna terrestre y lacustre boscosa en sitios de interior de bosque cercanos, como Lago Meliquina y Cueva Parque Diana, datados entre 2.300 y 480 años AP (Pérez \& Smith, 2008; Pérez \& Batres, 2008; Pérez et al. 2008); incluso del sector occidental cordillerano como Alero Marifilo 1 (Velásquez \& Adán, 2002; Adán \& Mera, 2011) y Alero Cabeza de Indio (Toro, 2012), entre otros; y que los diferencia de otros conjuntos óseos procedentes de sitios transicionales o de borde de bosque y de la estepa patagónica (Pérez, 2010; Fernández et al. 2016).

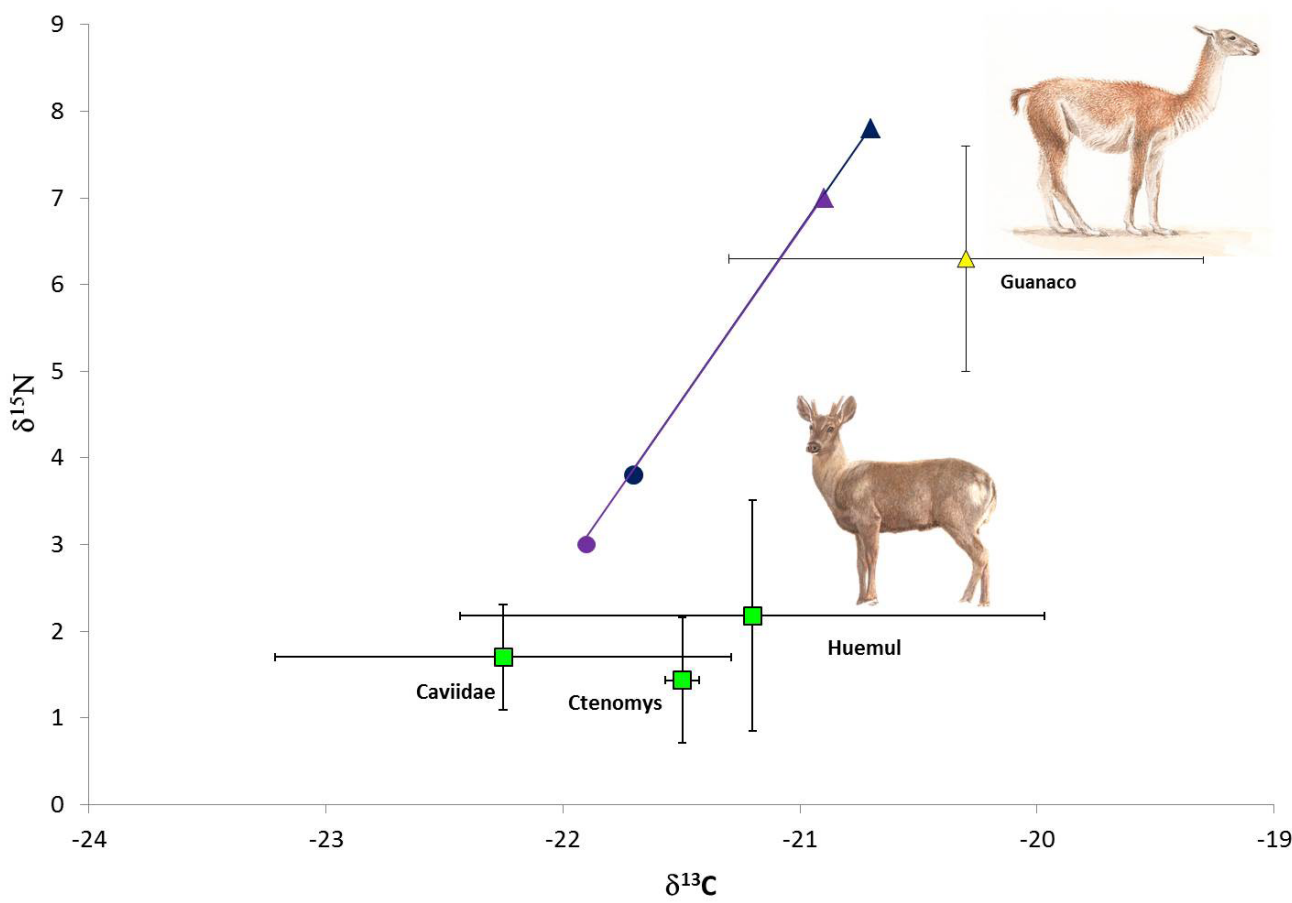

Fig. 3. Valores en restos humanos comparados con ecología isotópica. Triángulos registran valores $\delta{ }^{13} \mathrm{C}$ y $\delta{ }^{15} \mathrm{~N}$, mientras que los círculos las dietas estimadas a partir de considerar la discriminación isotópica. Izquierda inferior huemul y derecha superior guanaco. 


\section{CONSIDERACIONES FINALES}

Las implicancias de estos resultados se plantean a tres niveles diferentes. Primero, a nivel de la región, las muestras humanas analizadas reflejan la importancia del bosque y sus recursos en el último milenio en el sector meridional de Neuquén. Esto es coincidente con la evidencia zooarqueológica recuperada en estos sitios arqueológicos. Segundo, respecto a los modelos de uso de bosque en Patagonia Norte, los resultados aquí alcanzados vuelven a reforzar la idea de poblaciones humanas explotando de manera regular y permanente el bosque y sus recursos en la región andina oriental de Patagonia septentrional. Tercero, en relación a la metodología, nuevamente se ha puesto en valor la utilidad de los isótopos estables del carbono y nitrógeno para diferenciar las dietas terrestres de las poblaciones humanas del área andina de Patagonia.

\section{AGRADECIMIENTOS}

Este trabajo fue financiado por los subsidios a PICT 2011-1738 (ANPCyT), PI04173 (UNCo), PICT V 2014-1558 (ANPCyT) y la Honorable Legislatura de la Provincia de Neuquén. Agradecemos el apoyo de la Administración de Parques Nacionales (APNN ${ }^{\circ}$ 834) y de la Comunidad Mapuche Curruhuinca por su Consentimiento Previo, Libre e Informado. Al Diputado Guillermo Carnagui y al Sr. Vicegobernador Rolando Figueroa por su interés y apoyo. A los revisores anónimos del texto por sus constructivas sugerencias $y$ comentarios.

\section{BIBLIOGRAFÍA}

Adán, L., \& Mera, R. (2011). Variabilidad interna en el Alfarero Temprano del centro-sur de Chile: el Complejo Pitrén en el valle central del Cautín y el sector lacustre andino. Chungará, Revista de Antropología Chilena, 43(1), 3-23.

Adán, L., García, C., \& Mera, R. (2010). La Tradición Arqueológica de los Bosques Templados y su estudio en la región lacustre cordillerana de las regiones IX y XIV (Centro Sur de Chile). Sociedad Chilena de Arqueología (Eds.), Actas y Memorias del XVII Congreso Nacional de Arqueología Chilena, (pp. 1461-1471). Valdivia:
Sociedad Chilena de Arqueología.

Adán, L., Mera, R., Navarro, X., Campbell, R., Quiroz, D., \& Sánchez, M. (2016). Historia prehispánica en la región centro sur de Chile: Cazadores-recolectores holocénicos y comunidades alfareras (ca. 10.000 años a.C. hasta 1.550 d.C.). En F. Falabella, M. Uribe, L. Sanhueza, C. Aldunate \& J. Hidalgo (Eds.), Prehistoria en Chile. Desde sus primeros habitantes hasta los Incas (pp. 401-442). Santiago de Chile: Sociedad Chilena de Arqueología.

Aldunate, C. (1989). Estadio Alfarero en el sur de Chile. En J. Hidalgo, V. Schiappacasse, H. Niemeyer, C. Aldunate \& I. Solimano (Eds.), Culturas de Chile. Prehistoria (pp. 329-348). Santiago de Chile: Editorial Andrés Bello.

Aldunate, C. (1996). Mapuche: Gente de la Tierra. En J. Hidalgo, V. Schiappacasse, H. Niemeyer, C. Aldunate \& P. Mege, (Eds.), Etnografía. Serie Culturas de Chile (pp. 111-134). Santiago de Chile. Editorial Andrés Bello.

Aldunate, C., \& Villagrán, C. (1992). Recolectores de los bosques templados del cono-sur americano. En W. de Moesbach (Ed.), Botánica Indígena de Chile (pp. 2338). Santiago de Chile: Editorial Andrés Bello.

Arrigoni, G., \& Fernández, P. (2004). Los restos óseos del alero del Sendero de Interpretación (PN Los Alerces, Prov. del Chubut): Integridad, resolución y aprovechamiento de los recursos faunísticos del bosque. En Civalero, M.T., Fernández, P. M., \& Guraieb, A. G. (Comp.), Contra viento y marea. Arqueología de Patagonia (pp. 403-416). Buenos Aires: Instituto Nacional de Antropología y Pensamiento Latinoamericano.

Armesto, J., León-Lobos, P., \& Kalin, M. (1996). Los bosques templados del sur de Chile y Argentina: una isla biogeográfica. En J. Armesto, C. Villagrán \& M. Kalin (Eds.), Ecología de los Bosques Nativos de Chile (pp. 23-28). Santiago de Chile: Editorial Universitaria.

Barberena, R. (2002). Los límites del mar. Isótopos estables en Patagonia meridional. Buenos Aires: Sociedad Argentina de Antropología (SAA).

Barberena, R., Méndez, C., Mena, C. F., \& Reyes, O. (2011). "Endangered species, archaeology, and stable isotopes: huemul (Hippocamelus bisulcus) isotopic ecology in central western Patagonia (South America)". Journal of Archaeological Science, 38, 2313-2323.

Barberena, R., Tessone, A., Quiroga, M. N., Gordón, F., Llano, C., Gasco, A., Paiva, J., \& Ugan, A. (2018). Guanacos y Ecología Isotópica en el Norte del Neuquén: El Registro de Cueva Huenul 1. Revista del Museo de 
Antropología, 11, 7 -14.

Bellelli, C., Carballido, M., \& Stern, C. (2018). Obsidianas en el bosque: determinación geoquímica de artefactos arqueológicos del S-O de Río Negro y N-O de Chubut (Patagonia, Argentina). Chungará Revista de Antropología Chilena, 50(2), 201-216.

Borrero, L. A. (2005). The Archaeology of the Patagonian Deserts. Hunter-Gatherers in a Cold Desert. En P. Veth, P. Smith \& P. Hiscock (Eds.), Desert Peoples. Archaeological Perspectives, (pp. 142-158). Oxford: Blackwell Publishing.

Borrero, L., \& Barberena, R. (2006). Hunter-gatherer home range and marine resources. Current Anthropology, 47, 855- 867.

Campbell, R., Santana-Sagredo, F., de la Fuente, C., Moraga, M., Sánchez, M., \& Willerslev, E. (2017). El aporte de los isótopos estables a los estudios arqueológicos y ecológicos del Sur de Chile: El caso de Isla Mocha. Primer Simposio Nacional de isótopos estables, SIASIM 2017, Santiago, Chile.

Donoso, C. (1993). Bosques templados de Chile y Argentina. Variación, estructura y dinámica. Santiago de Chile: Editorial Universitaria.

Fernández, P. M., \& Tessone, A. (2014). Modos de Ocupación del Bosque Patagónico de la Vertiente Oriental de Los Andes: Aportes desde la Ecología Isotópica. Revista Chilena de Antropología, 30, 83-89.

Fernández, P. M., Calatayud, M. C., Bellelli, C., \& Podestá, M. (2013). Tiempo de cazadores. Cronología de las ocupaciones humanas en el valle del río Manso Inferior (Río Negro). En F. A. Zangrando, R. Barberena, A. Gil, G. Neme, G. Giardina, L. Luna, C. Otaola, S. Paulides, L. Salgán \& A. Tívoli (Comps.), Tendencias teóricometodológicas y casos de estudio en arqueología de la Patagonia, (pp. 167-175). Buenos Aires.

Fernández, P. M., Calatayud, M. C., Bellelli, C., Podestá, M., \& Scheinshon, V. (2011). Marcas en la piedra, huellas en la tierra. El poblamiento del bosque del suroeste de Río Negro-Noroeste de Chubut. Procesos históricos, transformaciones sociales y construcción de fronteras. Valverde, S., Maragliano, G., Impemba, M., \& Trentini, F. (coordinadores). Aproximaciones a las relaciones interétnicas. Estudio sobre Norpatagonia, Argentina y Labrador, Canadá, (pp. 195-221). UBA-FFyL.

Fernández, P. M., Cruz, I., Belardi, J. B., De Nigris, M., \& Muñoz, A. S. (2016). La explotación de huemul (Hippocamelus bisulcus, Molina 1782) en la Patagonia a lo largo del Holoceno. Magallania, 44(1), 187-209.

Flensborg, G., Martínez, G., \& Tessone, A. (2018). First approach to the paleodiet of hunter-gatherers through stable isotopes ( $\delta 13 \mathrm{C}$ and $\delta 15 \mathrm{~N}$ ) in the eastern PampaPatagonia transition during the Middle Holocene. Journal of Archaeological Science Reports, 17, 571580.

Gallardo, F., \& Mege, P. (2012). Cuando los frutos no dejan ver el bosque. Boletín de la Sociedad Chilena de Arqueología, 41-42, 43-52.

Gordón, F., Pérez, I., Hajduk, A., Lezcano, M., \& Bernal, V. (2017). Dietary patterns in human populations from northwest Patagonia during Holocene: an approach using Binford's frames of reference and Bayesian isotope mixing models. Archaeological and Anthropological Sciences, DOI 10.1007/s12520-016-0459-0.

Hajduk, A., Albornoz, A., \& Lezcano, M. (2008). Nuevos pasos en pos de los primeros barilochenses. Arqueología del Parque Nacional Nahuel Huapi. En C. Vázquez \& O. M. Palacios (Eds.). Patrimonio Cultural: la gestión, el arte, la arqueología y las ciencias exactas aplicadas, (pp. 175-194), Comisión Nacional de Energía Atómica. Argentina.

Lezcano, M. J., Hajduk, A., \& Albornoz, A. M. (2010). El menú a la carta en el bosque ¿entrada o plato fuerte?: una perspectiva comparada desde la zooarqueología del sitio El Trébol (lago Nahuel Huapi, Pcia. de Río Negro). En M. A. Gutiérrez, De Nigris, M., Fernández, P. M., Giardina, M., Gil, A. F., Izeta, A., Neme, G., \& H. D. Yacobaccio (Eds.), Zooarqueología a principios del siglo XXI: aportes teóricos, metodológicos y casos de estudio, pp. 243-257. Buenos Aires: Ediciones del Espinillo.

Navarro Harris, X., Dillehay, T. D. \& Adán Alfaro, L. (2011). Experiencias adaptativas tempranas de vida alfarera en el sector lacustre cordillerano de Villarrica. La ocupación del sitio Pucón 6 (IX Región). Cazadores Recolectores del Cono Sur, 4, 59-76.

Paruelo, J. M., Beltrán, A., Jobbágy, E., Sala, O. E., \& Golluscio, R. A. (1998). The climate of Patagonia: general patterns and controls on biotic processes. Ecología Austral, 8, 85-101.

Pérez, A. E. (2010). La Localidad Arqueológica "Lago Meliquina", Dto. Lácar, Neuquén. El registro arqueológico del interior y borde de bosque en Norpatagonia. En Sociedad Chilena de Arqueología (Eds.), Actas y Memorias del XVII Congreso Nacional de Arqueología Chilena, (pp. 1515-1528). Valdivia.

Pérez, A. E. (2016). El registro arqueológico de la Cuenca Binacional del río Valdivia. La integración de su fuente, el lago Lácar, sector oriental cordillerano. 
En M. A. Nicoletti, A. Núñez \& P. Núñez (Eds.), Araucanía-Norpatagonia: expresiones materiales $y$ representaciones de prácticas, político-económicas y socio-culturales, (pp. 141-187). 1ra. ed. Viedma. Universidad Nacional de Río Negro; San Carlos de Bariloche: IIDyPCa - Instituto de Investigaciones en Diversidad Cultural y Procesos de Cambio.

Pérez, A. E. (2018a). La ocupación del bosque meridional neuquino durante la Anomalía Climática Medieval (800 - 1400 DC). Revista Arqueología, 24(2), 297-301.

Pérez, A. E. (2018b). Tecnología cerámica de Los Radales 1. Período Alfarero Tardío (El Vergel), sector oriental cordillerano de la cuenca valdiviana (Neuquén, Patagonia Argentina). Arqueología Iberoamericana, 39, 29-35.

Pérez, A. E., \& Smith, M. (2008). Eficiencia depredadora y sistema de asentamiento en el bosque norpatagónico. La Localidad Arqueológica Meliquina, Parque Nacional Lanín, Neuquén. Las Ciencias. Revista Científica de la Universidad Maimónides, 1, 67-78.

Pérez, A. E., \& Batres, D. (2008). Los otros cazadores. La explotación de cérvidos en la Localidad Arqueológica Meliquina, Parque Nacional Lanín, República Argentina. En J.C. Diez (Ed.), Zooarqueología Hoy. Encuentros Hispano-Argentinos, (pp. 89-108). Universidad de Burgos.

Pérez, A. E., \& Reyes Álvarez, V. (2009). Técnica improntas de hojas. Algunas reflexiones acerca de su novedoso registro en la vertiente occidental cordillerana. Magallania, 37(1), 113-132.

Pérez, A. E., Smith, M., \& Grillo, E. (2008). Implicancias tafonómicas de la composición faunística en la Localidad Arqueológica Meliquina, Parque Nacional Lanín, Pcia. de Neuquén, Argentina. Runa, 29, 79-99.
Pérez, A. E., Reyes Álvarez, V., \& Erra, G. (2013). Economías mixtas de la Patagonia Noroccidental Argentina y centro sur de Chile. En M. A. Nicoletti y P. Núñez (Comps.), Araucanía-Norpatagonia: la territorialidad en debate. Perspectivas ambientales, culturales, sociales, políticas y económicas, (pp. 119-136). IIDyPCa-UNRN, CONICET.

Rizzo, F. (2017). Sitio Acevedo 1: restos humanos en el bosque del noroeste de Patagonia (localidad de Río Pico, provincia del Chubut). Intersecciones en Antropología, 18, 103-112.

Skewes J. C., \& Guerra, D. E. (2016). Sobre árboles, volcanes y lagos: algunos giros ontológicos para comprender la geografía mapuche cordillerana del sur de Chile. Intersecciones en Antropología, 17, 63-76.

Tessone, A. (2010). Arqueología y Ecología Isotópica. Estudio de isótopos estables de restos humanos del Holoceno tardio en Patagonia Meridional. Tesis Inédita. Facultad de Filosofía y Letras, Universidad de Buenos Aires, Buenos Aires.

Toro, O. S. (2012). Ocupaciones prehispánicas tardías en los bosques templados del sur de Chile. Una aproximación desde el uso del espacio en reparos rocosos. Boletín de la Sociedad Chilena de Arqueología, 41-42, 11-28.

Velásquez, H., \& Adán, L. (2002). "Evidencias arqueofaunísticas del sitio alero Marifilo 1. Adaptación a los bosques templados de los sistemas lacustres cordilleranos del centro sur de Chile". Boletín de la Sociedad Chilena de Arqueología, 33-34, 27-35.

Zilio, L., Tessone, A., \& Hammond, H. (2018). Stable isotope ecology and human palaeodiet in the northern coast of Santa Cruz (Argentine Patagonia). International Journal of Osteoarchaeology, 28, 305-317. 